\title{
Study on Multispectral Stealth Composites
}

\author{
Rongwang $\mathrm{Guo}^{1, \mathrm{a}^{*}}$, Liangyan Jiang ${ }^{1, \mathrm{~b},}$, Wannian $\mathrm{Zhu}^{1, \mathrm{c}}$ \\ ${ }^{1}$ Institute of engineers, Jiangsu Xuzhou,221004, China \\ a'Guo_rw@163.com, biang_ly@163.com, 'hu_wn@163.com
}

Keywords: Multispectral wave, Composites; Stealth technology.

\begin{abstract}
In this study, the authors use the infrared stealth theory of phase change materials into development of multispectral stealth composites. The study results showed that we can form a kind of hierarchical structured multispectral stealth composites, which can achieve good multispectral stealth effects in visible light and thermal infrared, radar, electromagnetic and laser, acoustic band etc. through technical processing on base material and phase change materials.
\end{abstract}

\section{Introduction}

With the rapid development of reconnaissance and detection technology and the use of precise-guidance weapons, "found means destroy" becomes a reality. So technology of target stealth becomes particularly important. Generally speaking, targets gain stealth performance by design of their appearance, structure and material to reduce target visible light, infrared, radar, electromagnetic, laser and acoustic characteristics of the signal. Among them, Stealth material is the foundation, which is also the key of stealth technology and the focus of military research in many countries. Modern reconnaissance and detection technology is combination of a variety of methods, which determines it will develop a direction towards multispectral stealth technology.

\section{Options of stealth composites}

Presently, research on single- spectrum stealth material and double-spectrum stealth material has already had many mature technology, but stealth material is mainly painted on targets, which would make it difficult to implement multispectral stealth. While direct application of structural stealth composites to targets can use stealth composites of various spectrum to incorporate and integrate in order to implement multispectral stealth.

Resin matrix composite (also known as glass fiber reinforced plastic) is chosen for convenient integration of stealth material as matrix material, which has a low density, high intensity, good transmission of waves and is economical, practical and easy for production, and can also incorporate with other stealth materials in forming process ${ }^{[1]}$.

At optical spectrum, three-color camouflage coating is used on the surface of targets to control brightness and chroma; in radar spectrum, radar absorbing material (RAM), such as nano graphite and ferrite, is mixed into the matrix material to absorb the radar wave transmitting into the matrix material; for laser stealth, laser stealth coating is applied to make it have a low reflectivity for laser, using rough surface to scatter laser at the same time to reduce the echo; for electromagnetic radiation of targets, high dense metal mesh can be used to shield itself own electromagnetic radiation, which also make targets invulnerable to external electromagnetic radiation; for the strong noise of most active targets, sound absorption and insulation materials can be used for shielding, which also have the effect of heat insulation; Active targets have strong infrared radiation, for which heat insulation methods must be used to shield heat with high reflectivity materials applied on the internal surface, such as aluminum foil; at the same time, thermal infrared camouflage coating can be used on the surface of targets to segment their infrared images, and phase change material (PCM) can be used to control the surface temperature of stealth composites. 


\section{Infrared stealth theory of phase change materials}

Phase change materials have a large specific heat capacity and will stay a relatively constant temperature during phase transition. So PCM can be used in composite material to control its thermal inertia and surface temperature, making its temperature cool and rise in consistent with the background or forming different temperature distribution on surface, to realize the effect of infrared fusion or segmentation with the background, the principle is as shown:

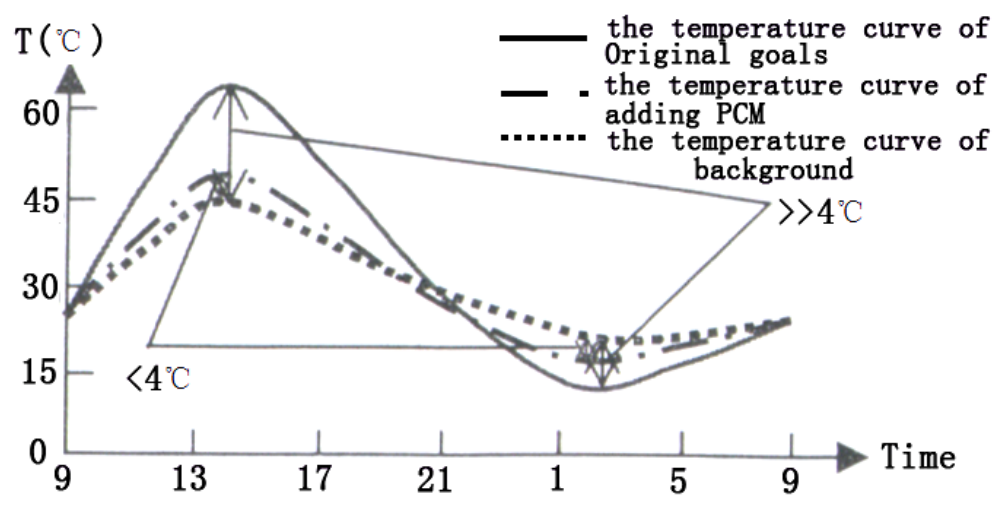

Fig.1 PCM infrared fusion mechanism

As is shown in figure 1, the surface of ordinary target differs greatly from that of the background in heat capacity, so they have great difference as the temperature changes, which makes them easy to be detected and attacked by enemy. While in the process of heating up ${ }^{[2]}$, PCMs absorb heat through phase change so as to inhibit the rise of targets temperature; In cooling process, the PCMs release heat to slow the process of cooling, thus making the temperature of the target in consistent with background, in which PCMs have played an important role in "cutting peak and filling valley ".

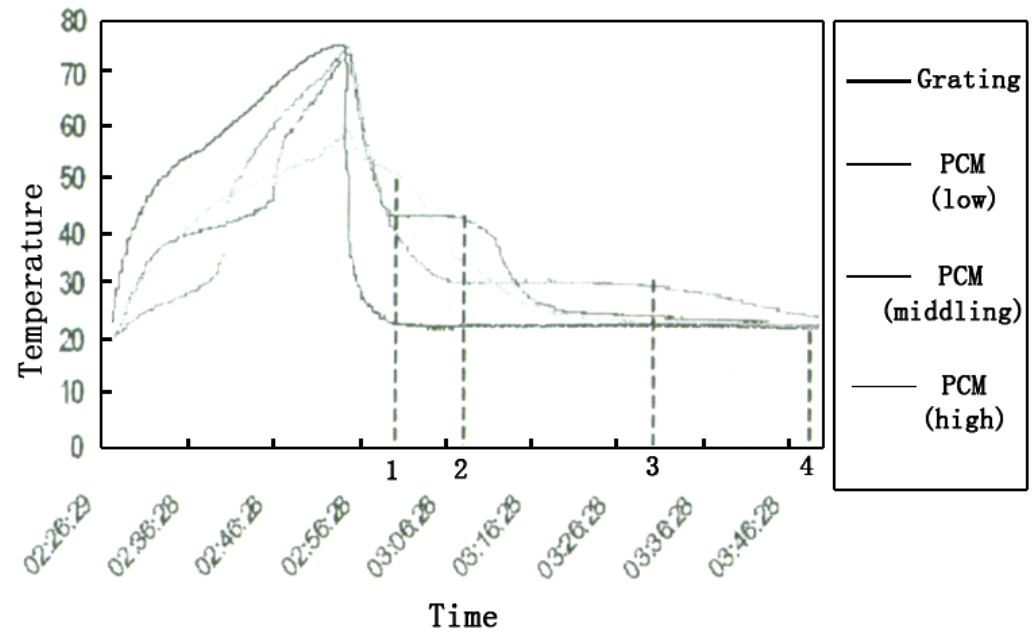

Fig.2 PCM infrared segmentation mechanism

As is shown in figure 2, PCMs with different phase change points are applied on the surface of targets. In the process of cooling and heating up, the surface of the target will present different temperature and the temperature distribution changes over time, which realize the effect of infrared segmentation.

The amount of PCMs is mainly determined by the intensity of solar radiation, while several elements must be taken into account such as the surface strength of actual targets, appearance size and space structure. The usage of materials amount has a limitation which means PCMs can't be applied according to the maximum. So methods of external incentives can be considered, for example, semiconductor refrigeration is used for the control of medium temperature to heat or cool PCMs by 
heat transfer structure, expand the phase change time of PCMs and finally realize all-weather infrared stealth.

\section{Integration of multispectral stealth composites}

Integration and compatibility of various spectrum stealth composites is key to the research on multispectral stealth composites. Layered matrix is available for stealth materials in order to realize multi-layered structural multispectral stealth composites.

At present, compatibility between visible light, infrared and laser is mainly achieved by using coating. For example, optical and infrared coating can achieve compatibility of visible light and infrared, the compatibility between $10.6 \mu \mathrm{m}$ laser and $8-14 \mu \mathrm{m}$ infrared spectrum can be achieved by the drilling method. However, the match between infrared coating and PCMs, radar stealth and electromagnetic stealth, and the compatibility between PCMs, matrix materials and RAMs should be solved.

Matching of thermal infrared coating and PCMs: PCMs and coating are applied for infrared segmentation as thermal infrared coating may lose segmentation effect when temperature of targets surface becomes high. PCM microcapsules are applied in matrix materials by means of mixing or sandwiching method to form PCMs. Infrared coating with the same infrared emissivity is painted on surface of the same matrix materials. The area with high phase change temperature should be painted with high infrared emissivity coating, and the same for low phase temperature area. Thus, when PCMs with high phase transition point change, surface temperature and emissivity are both high, which means infrared radiation is enhanced; when PCMs with low phase transition point change, surface temperature and emissivity are both low, which means infrared radiation is abated. In this way, infrared segmentation of targets surface is more apparent and the infrared stealth effect is enhanced ${ }^{[3]}$.

Compatibility among PCMs, matrix materials and RAMs: PCMs used for infrared stealth are usually paraffin material, which has no stimulating smell ,no corrosion, and has a large heat capacity, but its thermal conductivity is poor. Paraffin PCMs can fill in the sandwich of matrix material to control its surface temperature. As matrix and PCMs are all transparent materials, therefore RAMs can be mixed into matrix and PCMs, which can absorb radar wave transmitted into the matrix and PCMs. At the same time, if absorbing material has a good thermal conductivity, such as graphite, it can increase the thermal conductivity of matrix and PCM, which can realize a more rapid and accurate control on matrix surface temperature.

Matching of radar stealth and electromagnetic stealth: High dense metal mesh can be used to shield target electromagnetic radiation, metal mesh at the same time can block radar wave transmitting to the surface of targets, then protecting targets from radar detection and recognition. Radar wave is absorbed by absorbing materials for the second time after spreading through the matrix and PCMs, finally only a small amount of radar echoes transmit out from the surface of stealth material so as to achieve the purpose of radar stealth. As is shown in figure 3:

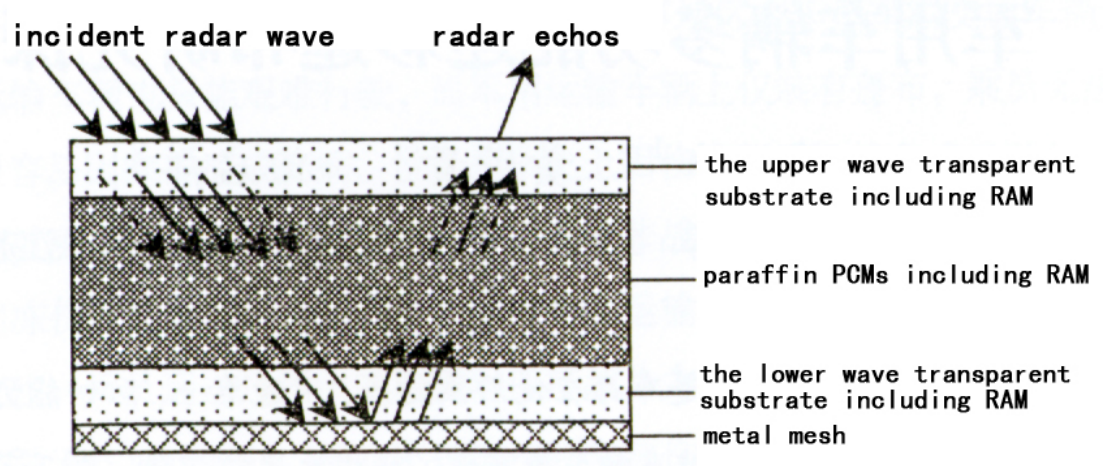

Fig.3 The schematic diagram of radar absorbing wave 


\section{Conclusion}

By painting visible light, thermal infrared and microwave compatibility coating on the surface of matrix materials(resin matrix composites), filling matrix sandwich with PCMs, mixing RAMs of good thermal conductivity into matrix and PCMs, compositing high dense metal mesh, heat insulation materials, sound-absorbing materials and high- reflectivity materials, a kind of hierarchical structured multispectral stealth composites can be developed to make targets using this composites obtain good stealth performance in visible light, infrared, radar, electromagnetic ,laser and acoustic vibration.

\section{References}

[1] Zhong H, Li Z.L. Stealth technology[M].Beijing: National defense industry press 1999:98-102.

[2] Wang Z. R, Sun X.Q, Yu X.B. Analysis of several contradiction problems in multi-band stealth compatibility. Modern defense technology.2004.32 (6):32-35.

[3] Zhang Z.B, Shen W.D, Song S.H. Application of Resin Matrix Composite in Stealth Technique. Electro-optic Technology Application. 2005(8):23-25. 